\title{
La place des firmes dans la tradition et l'actualité géopolitiques
}

The place of firms in geopolitical tradition and issues

Der Platz der Firmen in der geopolitischen Tradition und der geopolitischen

Aktualität

\section{Stéphane Rosière}

\section{CpenEdition}

\section{Journals}

Édition électronique

URL : http://journals.openedition.org/rge/2942

DOI : $10.4000 /$ rge.2942

ISSN : 2108-6478

Éditeur

Association des géographes de l'Est

Édition imprimée

Date de publication : 31 octobre 2010

ISSN : 0035-3213

Référence électronique

Stéphane Rosière, "La place des firmes dans la tradition et l'actualité géopolitiques », Revue Géographique de l'Est [En ligne], vol. 50 / 1-2 | 2010, mis en ligne le 29 septembre 2011, consulté le 08 septembre 2020. URL : http://journals.openedition.org/rge/2942 ; DOI : https://doi.org/10.4000/rge. 2942

Ce document a été généré automatiquement le 8 septembre 2020.

Tous droits réservés 


\title{
La place des firmes dans la tradition et l'actualité géopolitiques
}

\author{
The place of firms in geopolitical tradition and issues \\ Der Platz der Firmen in der geopolitischen Tradition und der geopolitischen \\ Aktualität
}

Stéphane Rosière

\section{Introduction}

1 Pensée historiquement et quantitativement (ce qui ne veut pas dire uniquement) comme une étude des relations entre les États, et se distinguant mal de ce point de vue de l'approche «réaliste» des Relations internationales, la géopolitique classique accorde une place marginale aux acteurs économiques ${ }^{1}$. Seule la géoéconomie, formulée dans les années 1990, a clairement tendu à renverser les prismes et à se centrer sur l'économie et ses agents (les entreprises et surtout les firmes multinationales). La critical geopolitics anglo-saxonne, analyse des discours géopolitiques qui s'est largement développée depuis les années 1990, est elle aussi relativement centrée sur les discours des gouvernants. On le voit, la place des entreprises et plus précisément des firmes - définit comme « une entreprise, plutôt de grande taille 》 (Brunet, 1993, p.217) - dans la géopolitique apparaît marginale, ce qui ne veut pas dire inexistante. Cette marginalité soulève de nombreuses questions épistémologiques.

2 Notre papier est structuré en une réflexion sur les développements historiques de la discipline qui sera d'abord menée (1e partie), puis nous soulignerons le poids croissant des firmes, notamment multinationales, et des acteurs financiers dans le monde contemporain marqué par la création d'une "superclasse » oligopolistique (un petit groupe dominant et en position de monopole). Cette situation pose la question d'une lutte pour l'hégémonie entre les firmes et l'État, lutte qui, concomitamment, a des implications sur la géopolitique en tant que discipline scientifique (2e partie). 


\section{La place des firmes dans la tradition géopolitique}

3 La géopolitique est une démarche praxéologique (ayant pour but d'âtre appliquée ${ }^{2}$ ) qui, d'un point de vue historique, a d'abord été pensée comme une étude des relations entre les États tenant compte des facteurs géographiques. De nombreuses définitions sont fondées sur ce paradigme : pour citer Pierre-Marie Gallois - parmi d'autres - elle serait donc une "étude des relations qui existent entre la conduite d'une politique de puissance portée sur le plan international et le cadre géographique dans lequel elle s'exerce» (Gallois, 1990, p. 37).

\section{A. La démarche géoréaliste : l'État au centre}

Suivant cette approche toujours très prégnante, le géopolitique se distingue mal des Relations internationales (l'apparition des deux disciplines étant par ailleurs presque concomitante). Il est vrai que, comme pour P.-M. Gallois, beaucoup d'auteurs qui pratiquent cette géopolitique ne sont pas des géographes. Peut-être les géopoliticiens utilisent-ils plus de cartes que les spécialistes de Relations Internationales, néanmoins, géopolitique et école réaliste des Relations Internationales se trouvent amalgamées en une matrice unique : "géoréaliste » selon l'expression judicieuse de Frédéric Lasserre et Emmanuel Gonon (2008).

5 L'ensemble de la géopolitique classique - qui va de Friedrich Ratzel à la fin du XIXe siècle jusqu'à la Seconde Guerre mondiale et inclue Karl Haushofer, Halford MacKinder (1904), et Nicholas Spykman (1944) - est "géoréaliste». Elle accorde une place marginale aux acteurs économiques, mais pas à l'économie - on soulignera quand même l'exception d'Isaïah Bowman (1928) qui lui accorde une place plus importante.

Parmi les fondateurs, Friedrich Ratzel (qui écrit la première géographie politique en 1897) insiste beaucoup sur la circulation et l'économie ${ }^{3}$. Bien sûr, sa vision de l'économie est aujourd'hui désuète (lien puissance économique - sol, dans le droit fil de son analyse de l'État comme " organisme » lié au sol - chapitre 1 de la première partie de sa Géographie politique), mais il met cependant en évidence des formes de puissance non territoriales : «la politique des puissances commerciales [passe] par une dépréciation du sol, auquel elles préfèrent les biens mobiles, faciles à acquérir et à échanger (...).» (Ratzel, p. 77). Cependant, et parce qu'il privilégie « l'étude comparative des relations qu'entretiennent l'État et le sol » (Ratzel, 1987, p. 2), pour lui c'est la mise en valeur du sol qui confère la richesse (Bergevin, 1989). "Plus l'individu labourant, semant, récoltant, incorpore de travail dans sa portion de terre, et plus celle-ci prend à ses yeux de prix ; plus il s'y attache solidement, et plus il en augmente la valeur politique, c'est-à-dire plus les liens se resserrent, à travers tous ces maillons intermédiaires, entre la collectivité et l'État " (Ratzel, 1987, p. 59). Dans cette vision, l'espace produit de l'économique qui génère du politique. Les liens économiques consubstantiels à la mise en valeur de la surface terrestre précèdent les rapports politiques car «... l'économie est plus proche du sol que la politique » (p. 59).

7 Friedrich Ratzel n'est pas indifférent à la structure économique du monde, et il en devine même l'importance croissante : «Le commerce mondial tend à transformer toute la terre en un organisme économique unique, au sein duquel les peuples et les pays ne seront que des organes plus ou moins subordonnés. Combien de courants commerciaux convergent déjà sur Londres! Il faut à un peuple une extrême énergie et beaucoup de ténacité pour conserver son indépendance culturelle et politique au milieu de ce mouvement de concentration» (p. 28). 
Ainsi, sa mise en exergue de la dimension économique de l'espace n'intègre pas réellement les firmes, elle les gomme par l'échelle à laquelle elle l'envisage alors que ses considérations tournent plus autour des peuples et des États - ce qui est vrai chez tous les « géoréalistes » (que nous ne pourrons tous citer ici).

\section{B. La remise en cause de l'approche « géoréaliste »}

8 La contestation de l'approche "géoréaliste» a pris la forme d'une étude des interactions «transnationales» notamment chez les analystes des Relations internationales (RI) dès les années 1960, comme Arnold Wolfers (1892-1968). Ces auteurs postulaient nettement la pluralité des acteurs dans le champ des RI. «Une théorie qui ne se préoccupe que des Etats comme acteurs est irréaliste, avant tout parce qu'elle néglige la prise en compte des individus comme acteurs à part entière des relations internationales, ensuite parce qu'elle ne considère pas les autres acteurs que sont les organisations internationales, les groupes organisés, etc "(Wolfers, 1962, p.24). Arnold Wolfers ne mettait pas encore, en 1962, nettement l'accent sur les firmes, mais par sa mise en avant de «l'individualisme méthodologique », la rupture épistémologique avec l'interprétation statocentrée du " géoréalisme » était bien consommée.

9 Les théoriciens des relations "transnationales» se focalisent sur les processus d'intégration économique et politique à l'échelle de la planète et la théorie de l'interdépendance, proposée par Joseph Nye (né en 1937) et Robert Keohane (né en 1941) confirme cette évolution. Le paradigme de l'interdépendance en RI met l'accent sur l'interdépendance et la coopération en considérant que les relations internationales contemporaines ne correspondent plus au modèle conflictuel et interétatique des théories réalistes. Robert Keohane et Joseph Nye publient en 1977 Power and Interdependance où ils soulignent l'érosion des cadres préétablis d'autorité fondés sur la puissance et, corrélativement, le rôle de plus en plus décisif des grandes firmes multinationales (mais aussi des institutions internationales, des ONG ou des lobbys). L'interdépendance rend délicate la détermination de "l'intérêt national » tout comme elle accentue d'ailleurs la fongibilité entre les structures et les acteurs...

On retrouve plus ou moins cette évolution chez les géographes états-uniens, des années 1960 , et notamment chez les «fonctionnalistes " anglo-saxons. Parmi eux, Norman Pounds (1963) révisent aussi les visions trop «statocentrées » des aînés. La vision de la géographie politique des «fonctionnalistes » évolue, le rôle de l'économie est souligné même si n'est pas encore au centre de leur préoccupation, elle apparaît néanmoins... Dans sa Political Geography, Norman Pounds consacre ainsi des chapitres aux questions économiques que sont: "Resources and Power" (1963, pp.144-170), "The Foreign Geography of Political Trade» (pp.249-277) ou «The Underdeveloped World» (pp. 357-386).

11 Des remises en cause importantes sont aussi venues de la géographie radicale et de la géographie critique. Apparue dans les années 1970, l'approche radical exprime une interprétation marxiste des sciences et de la société alors que l'adjectif critical renvoie à une démarche plus attentive aux conditions dans lesquelles les théories et représentations sont formulées. Plus post-moderne (notamment par son questionnement sur les notions de réalité et d'objectivité), l'approche critique met l'accent sur la diversité des points de vue et des représentations. 
12 En France, on peut considérer l'école lacostienne de géopolitique, apparue dans les années 1970, tout à la fois comme une émanation du courant critical (ou critique en français) notamment par l'étude attentive des représentations et radical par sa remise en cause de la grille de lecture "réaliste ». Dans une perspective moins statocentrée que la géopolitique classique, Yves Lacoste proposait une révolution " copernicienne " bienvenue en pensant la géopolitique comme étude des "rivalités de pouvoir» (Lacoste, 1993). Ce positionnement novateur évitait le "piège étatique " dénoncé par Arnold Wolfers dans les Relations internationales, sinon le « piège territorial » dénoncé par John Agnew (1994) dans la géographie politique - les deux auteurs dénonçant la trop grande attention portée à l'État et à ses frontières. Surtout, il permettait d'ouvrir la géopolitique à de multiples acteurs - nous serions tenté de dire, à tous les acteurs de la géographie. Par sa définition radicalement nouvelle, Y. Lacoste participait donc au dépassement du paradigme étatique (symbolisé par les très nombreuses définitions de la géopolitique la considérant comme l'analyse de la politique des États relative à leur " géographie »).

Dans son versant post-moderne, ou critique, la géopolitique critique (critical geopolitics) anglo-saxonne est largement devenue une analyse des discours et représentations. Elle serait même une refondation de la géopolitique par l'analyse du discours ${ }^{5}$. Pour Gearoid O'Tuathail la géopolitique critique est une analyse de l'impérialisme, et de ses discours (O'Tuathail \& Agnew, 1992 ; O'Tuathail, 1993, 1996). G. O'Tuathail a montré comment, à la fin des années 1980, se pose pour les Etats-Unis le problème d'un certain déclin (que Paul Kennedy exprimait aussi en 1987) relatif au poids économique croissant du Japon (1993), ce déclin suscite alors un débat que l'auteur considère comme géopolitique et qui est avant tout un débat économique. Ces réflexions géoéconomiques soulignent la mutation alors en cours dans la géopolitique.

14 D'une façon générale, depuis les années 1990, la géopolitique considère avec plus d'attention les acteurs économiques. Cependant, l'économie, ou le développement, n'est pas un chapitre du Political Geography Handbook paru assez récemment et relativement exhaustif (Cox \& alii, 2008) et le mot firme n'intègre pas l'index de l'ouvrage. C'est dire que la place de l'économie et des firmes dans la doxa de la géographie politique n'est pas encore assurée et que des courants, mais aussi des inerties ou des fongibilités intellectuelles se distinguent aisément.

Il faut attendre les années 1990, l'accélération de la «libéralisation» du monde consécutive à l'effondrement du bloc soviétique - pour qu'une théorie propose une analyse de l'espace en fonction de hiérarchies clairement bouleversées: la géoéconomie. Celle-ci est lancée par Edward Luttwak (né en 1942) dans un article publié dans le National Interest en 1990 - mais soulignons quand même que celui-ci n'a pas non plus consacré d'ouvrage à ce thème.

16 Edward Luttwak souhaitait renverser les prismes géopolitiques et penser l'espace économiquement: "It is true, of course, that, under whatever name, 'geo-economics' has always been an important aspect of international life. (...) Now, however, as the relevance of military threats and military alliances wanes, geo-economic priorities and modalities are becoming dominant in state action » (Luttwak, 1990). Cette approche ne veut pas dire que les acteurs étatiques soient nécessairement oubliés; ainsi, comme le précise Pascal Lorot, "la géoéconomie est l'analyse des stratégies d'ordre économique - notamment commercial -, décidées par les États (...) » (2001, p.46). 
17 Le fait de considérer la géopolitique comme dépassée implique évidemment que l'on définisse cette discipline comme statocentrée (cf. définition de P.-M. Gallois, plus haut), une étude de l'État, ou des relations interétatiques - à ce moment-là, effectivement, la discipline est obsolète dans le contexte de mondialisation, mais cela n'est plus le cas avec la définition renouvelée d' Yves Lacoste mentionnée plus haut - voire et dans la même veine épistémologique celle que j'ai proposé dès 2001 en définissant la géopolitique comme "étude de l'espace comme enjeu» (2003). Rivalités de pouvoir ou enjeu n'impliquent aucun acteur exclusif et la translation de l'État vers les firmes ne serait qu'une variation à l'intérieur de la discipline, pas un changement de discipline.

\section{La mondialisation et la montée en puissance des firmes et des acteurs financiers}

18 Le «nouvel ordre mondial» des années 1990 est pensé et formulé comme un ordre économique, l'espace est d'abord analysé suivant des critères géoéconomiques. Le principe fondamental de cette pensée est la liberté d'entreprendre, doctrine absolue qui implique la liberté des échanges et la destruction des «obstacles» et autres restrictions aux échanges... autant de signes de la montée en puissance des firmes et des acteurs financiers en général.

Hors du champ des disciplines à "tropisme» spatial, rappelons Susan Strange (1923-1998), l'un des fondateurs de l'économie politique internationale (que nous pourrons comprendre aussi comme une mutation des relations internationales en phase avec le poids croissant des questions et des acteurs économiques), qui insiste sur le rôle nouveau et décisif des marchés financiers plus que sur les entreprises en tant que telles.

20 Dans Retreat of the State(mais aussi Rival States, Rival Firmes), S. Strange soulignait la montée en puissance de nouveaux acteurs comme les mafias, les assurances et les grandes compagnies d'expertise comptable (les Big Six), les bureaucraties internationales constituées par les organisations intergouvernementales, et les firmes multinationales (FMN). Ces dernières ont contribué à changer la nature de la compétition entre Etats ainsi que leur mode de fonctionnement. Pour Susan Strange, ce sont spécifiquement les FMN qui ont contribué à changer la nature de la compétition entre les Etats ainsi que leurs modes de fonctionnement, ce sont elles qui ont réussi à imposer la mondialisation « néolibérale ».

\section{A. Logique la mondialisation}

L'ordre économique et politique contemporain esquissé par les accords de BrettonWoods en 1944, et renforcé par l'écroulement du bloc soviétique en 1989, est fondé sur l'idée selon laquelle le monde pourrait être unifié et pacifié par le commerce (avec comme cadre Bretton-Woods puis un autre système, moins régulé, depuis la fin de la convertibilité du dollar en or le 15 août 1971). Notons le retour à une certaine régulation depuis la crise financière de 2008 -soulignons ainsi la réhabilitation de la « taxe Tobin " prônée par les dirigeants européens en 2009, bien que jugée hérétique avant la crise. 

(The Superclass en anglais), David Rothkopf (2009) s'y est essayé et a rappelé, entre autres, que « en 2007 (...), la production mondiale était estimée à 47000 milliards de dollars. La même année, les 250 plus grandes entreprises réalisaient un chiffre d'affaires supérieur à 14870 milliards de dollars, soit près d'un tiers de la production mondiale et un montant plus important que le PIB des Etats-Unis cette année-là (13 200 milliards) ou de l'Union européenne (13 740 milliards). A elles seules, les 100 premières entreprises réalisent 9720 milliards de \$, et les 5 premières - Wal-Mart, ExxonMobil, Royal Dutch Shell et General Motors - 1500 milliards soit plus que le PIB de n'importe quel pays, à l'exception des 7 plus riches d'entre eux. » (Rothkopf, 2009, p.50)

29 La comparaison entre les chiffres d'affaires des firmes et les PIB des États offrent effectivement des enseignements remarquables. Selon D. Rothkopf, « ExxonMobil dépasse l'Arabie saoudite (la $25^{e}$ plus riche nation du monde), Wal-Mart se situe entre l'Indonésie et la Pologne» (p.50-51), etc. Evidemment, c'est surtout par rapport à la majorité des pays pauvres que la comparaison est la plus criante. D. Rothkopf considère ainsi toutes les « entités » (État ou firme) dont le PIB ou le chiffre d'affaire dépasse les 50 millions de dollars et il en recense (en 2007 toujours) 166 dont 60 seulement sont des États (p.51), soit à peine un tiers du total. 
30 L'influence de ces grandes firmes vis-à-vis de leur environnement local, régional, national est énorme. Sans même parler de pays sont les "pantins» de certaines compagnies, ou des paradis fiscaux ont le rôle est d'accroître les marges des acteurs financiers transnationaux, cette influence se fait directement sentir sur les fournisseurs, qui dépendent de leurs commandes, et au-delà sur l'ensemble de la vie économique. Le nombre de leurs employés très important pour les plus grosses (Toyota comptait 316000 employés en 2008), leurs réserves financières, le réseau mondialisé qu'elles constituent les rendent aussi influentes que des États mais par d'autres canaux que ces derniers. Le lobbying est une de leurs armes décisives à Washington comme à Bruxelles. David Rothkopf rappelle que les 200 premières entreprises rassemblent (en 2007) « quelque 27000 milliards de dollars de chiffre d'affaire annuel et un capital de 103000 milliards. Elles emploient plus de 70 millions de travailleurs [et font vivre environ] 350 millions d'êtres humains. En réalité, ce chiffre est plus élevé encore si l'on prend en compte les emplois qu'elles génèrent chez leurs fournisseurs et sous-traitants. " David Rothkopf estime à un milliard le nombre d'individus dont l'existence dépend directement des décisions des grandes FMN.

31 On se demande désormais si les Etats ont encore du pouvoir face aux FMN : le poids économique de ces firmes est parfois énorme, leurs décisions stratégiques semblent ne pas pouvoir être contrôlées, ce qui fait apparaître les Etats comme impuissants à exercer une quelconque activité de contrôle. Ainsi, et logiquement, peut-on se demander si plus que des structures de plus en plus décisives, les firmes multinationales ne sont-elles pas devenus directement des rivales des États?

\section{Les firmes contre les États?}

La montée en puissance des firmes et des acteurs économiques pourrait être analysée au moins en tant qu'hypothèse - comme générant une lutte contre les États, une lutte pour l'hégémonie à l'échelle mondiale dont la marque est la déconstruction des normes westphaliennes et l'avènement d'un ordre marchand ou financier.

Cette hypothèse a été envisagée par Raoul Marc Jennar et Laurence Kalafatidès dans le cadre du processus mené par l'Organisation Mondiale du Commerce (OMC) de négociation de l'Accord général sur le commerce des services (AGCS ou GATS en anglais) négocié depuis 1995.

Depuis sa fondation en 1995, le rôle fondamental de l'OMC est de parvenir à supprimer toute forme de restriction à ces activités « marchandes », en les « libérant » des normes et lois adoptées par les États au nom de l'égalité des droits ou d'autres principes généraux. Dans cette perspective, le corpus législatif mondial est assimilé par l'OMC à une "entrave au commerce ». Comme on l'adit plus haut, les États sont, dans cette perspective, réduit à une gène "environnementale", des parasites institutionnels. Pour R. M. Jennar et L. Kalafatidès, l'OMC «prend clairement pour cible les fonctions régulatrices et redistributrices des pouvoirs publics et, si nécessaire, le contrôle démocratique là où il existe [et] vise in fine à substituer la souveraineté des marchés à la souveraineté et aux droits des peuples » (Jennar \& al., p.36), toutes les règles de l'OMC contraignent d'ailleurs les États bien plus que les firmes ou les entreprises...

35 Le processus de libéralisation des services lancé après la Seconde Guerre mondiale s'est accéléré avec la création de l'OMC en 1995, sous la pression des acteurs financiers américains (dont l'assureur AIG... nationalisé de facto en 2008 pour échapper à la 
faillite... selon David Hartridge, le directeur de la division du commerce des services de l'OMC). L'AGCS est le résultat d'un "art» du lobbying clairement mis en évidence incluant de puissants acteurs économiques nord-américains (TABD, etc.) mais aussi européens (UNICE, ERT, etc.) et au premier rang desquels les FMN et les acteurs financiers. L'intrication entre ces acteurs décisifs avec le Nord est profonde: la «Quad» qui dirige de facto l'organisation est constitué par le quatuor USA, Canada, UE et Japon.

L'objectif de l'AGCS est non seulement de libéraliser le commerce mais aussi les " services ", notion floue intégrant l'environnement, l'éducation, la santé, la culture et la liste est longue car l'OMC a répertorié 160 activités de services qui sont considérés comme des "marchés». Cette logique de marchandisation des services relève elle même d'une privatisation générale des modes de vie ainsi que d'une marchandisation du minéral, du végétal, de l'animal, de l'humain et de tout ce que l'humain peut produire -ce que l'on peut appeler, à tord, le néo-libéralisme en ce qu'il consiste en la dissémination des valeurs du marché à la politique, la vie intellectuelle, sinon la vie tout court. Les effets de l'AGCS sont d'obliger les États à créer un "environnement compétitif non faussé» entre entreprises publiques et privées ce qui se traduit soit par une diminution des subventions aux premières, soit par l'aménagement d'aides particulières pour les entreprises privées. Les subventions au niveau régional et communal sont aussi visées par l'AGCS qui joue le désengagement des collectivités à toutes les échelles.

In fine, selon Raoul Marc Jemmar et Laurence Kalafitidès, l'OMC aurait réussi à enserrer ses 153 États membres (en juillet 2008) « dans un étroit maillage de contraintes, d'obligations et de principes" (2007, p.71). Et ces contraintes ne sont pas chimériques. Pour ces auteurs, l'OMC est aujourd'hui «l'organisation internationale la plus puissante du monde [puisque] tout à la fois pouvoir législatif, pouvoir exécutif et pouvoir judiciaire, donc simultanément juge et partie dans les très vastes matières en sa compétence [absence de séparation des pouvoirs]. En deuxième lieu, l'OMC impose le respect des règles qu'elle gère. Chaque pays doit adapter ses lois (...) aux règles de l'OMC. » L'OMC peut enfin juger un État (notamment sur la base d'une plainte d'un autre État) et définir les sanctions encourues. Elle est donc à la fois juge et procureur.

Cette interprétation, évidemment contestée par les partisans de l'organisation, a au moins le mérite de pousser à l'extrême les logiques actuelles et d'esquisser un monde et une géopolitique profondément renouvelées dans laquelle les firmes et les acteurs financiers seraient devenus les vrais maîtres du jeu et les États des entités de moindre importance, vassalisées par les premières. Épistémologiquement, ce serait évidemment la disparition de la géopolitique (classique seulement), et fatalement le renforcement de la géoéconomie.

De ce point de vue, il importe de souligner que, parmi les firmes, les acteurs financiers se distinguent et semblent jouer désormais le rôle le plus important. Nous pouvons citer: les banques d'affaires et de dépôt (si tant est que la distinction est encore un sens), les compagnies d'assurances, les fonds (de pension, souverains, d'investissement, hedge fund, vautours, de capital-investissement, etc.) et les agences de notation, voilà grosso modo les firmes - que l'on pourrait qualifier de firmes à finalité financière - (FFF) qui se trouvent aujourd'hui en position de force - que l'on pense à Goldman Sachs...

En novembre 2007, près de 10000 hedge funds étaient opérationnels dans le monde, aux trois-quarts américains ou britanniques et géraient environ 1760 milliards USD (1 250 
milliards d'euros). En 2009, les deux tiers des hedge funds étaient localisés dans les îles Caïmans devenus de facto le paradis fiscal des multinationales...

\section{Conclusion}

41 financières) ou des acteurs économiques et financiers est assurément un des éléments clefs de la période contemporaine, elle participe à l'évolution des disciplines qui, comme la géopolitique, ont pour ambition de décrire et de théoriser l'espace mondial et ses acteurs.

La mise en place de formes de "gouvernance » (terme banalisé et pourtant loin d'être neutre qui évacue le "gouvernement») est aussi le produit d'un retrait consenti de l'État et par lui stimulé. Ce « retrait de l'État » est mis en œuvre dans un certain nombre d'organisations internationales et particulièrement dans l'Organisation mondiale du commerce $(\mathrm{OMC})$ qui tend à établir la suprématie des entreprises dans un monde où la chose publique est déconsidérée et dévalorisée. On a là une stratégie d'hégémonie orchestrée par une catégorie d'acteurs qui détient bien souvent les clefs du pouvoir étatique pour asseoir sa suprématie économique et vice-versa. Dans un cas central, celui de la Chine, le philosophe et politiste Liu Xiaobo (actuellement emprisonné, prix Nobel de la pais en 2010) avait montré dans l'une de ses études que plus de $90 \%$ des 20000 personnes les plus riches de Chine possèdent des liens familiaux avec de hauts responsables du gouvernement et du parti. Dans une autre perspective rappelons aussi que près de la moitié des fonds gérés par les hedges funds sont détenus par des investisseurs institutionnels (Inchauspé, 2010)... Le quotidien Le Monde avait, lui aussi, montré la consanguinité des conseils d'administration du CAC 40 (98 personnes détenant $43 \%$ des droits de vote des entreprises de l'indice) et leur proximité avec les gouvernements de la Ve république (Kahn, 2010). Ainsi, la fongibilité entre les domaines du " politique » et de l' " économique » doit être soulignée, elle constitue une donnée pérenne en termes épistémologiques. De ce fait, l'enjeu en termes d'analyse du ou des pouvoir(s) serait de se situer à la jointure de l'économique et du politique plutôt que strictement dans l'une ou dans l'autre de ces dimensions.

\section{BIBLIOGRAPHIE}

Agnew J., 1994, « The territorial trap: the geographical assumptions of international relations theory ", Review of International Political Economy, $\mathrm{n}^{\circ} 1 / 1$, pp.53-80.

Bergevin J., 1989, «A propos de la géographie politique : la parole est à Friedrich Ratzel », Cahiers de Géographie du Québec, vol. 33, n88, pp.59-66, consulté le 9 juin 2010, URL : http:// www.erudit.org/revue/cgq/1989/v33/n88/022000ar.pdf

Bowman I., 1928, The New World: Problems in Political geography, Yonkers-on-Hudson (NY) \& Chicago, World Book Company.

Revue Géographique de l'Est, vol. 50 / 1-2 | 2010 
Brunet R., 1993, Les mots de la géographie. Dictionnaire critique, Montpellier / Paris, RECLUS / La Documentation Française, 518 p.

Cox K., Low M., Robinson J., 2008, Handbook of Political Geography, Londres, Sage, 640 p.

Foucher M., 1991, Fronts et frontières, un tour du monde géopolitique, Paris, Fayard, 527 p.

Gallois P. M., 1990, Géopolitique. Les voies de la puissance, Paris, FEDN/Plon, 474 p.

Hibou B., 1999, La privatisation des États, Paris, Karthala, 398 p.

Inchauspé I., 2010, "L'union doit prendre les hedge funds à revers : interview de Michel Aglietta », Challenges, $\mathrm{n}^{\circ} 200,18$ février, p. 42

Jennar R.M., Kalafatides L., 2007, L'AGCS. Quand les États abdiquent face aux multinationales, Paris, éditions Raisons d'agir, $122 \mathrm{p}$.

Kahn A., 2010, « CAC 40 : la consanguinité des conseils d'administration perdure », Le Monde, 12 janvier.

Lacoste Y., Dictionnaire de géopolitique, Paris, Flammarion, 1 ère édition.

Lasserre F., Gonon E., 2008, Manuel de géopolitique. Enjeux de pouvoir sur des territoires, Paris, Armand Colin, Coll. «U », 347 p.

Lorot P., 2001, « La géoéconomie, nouvelle grammaire des rivalités internationales », L'Information géographique, Vol. 65, mars, p.43-52.

Luttwak E. N., 1990, « From Geopolitics to Geoeconomics: Logic of Conflict, Grammar of Commerce ", The National Interest, $\mathrm{n}^{\circ} 20$, summer, p. 17-23.

Mackinder H. J., 1904, « The geographical Pivot of History », The Geographical Journal, Vol. XXIII, $\mathrm{n}^{\circ} 4, \mathrm{p} .421-444$

Ó Tuathail G., Agnew J., 1992, « Geopolitics and Discourse : Practical Geopolitical Reasoning in American Foreign Policy », Political Geography Quarterly, Vol. 11, p.190-204.

Ó Tuathail G., 1993, «Japan as a Threat: Geo-Economic Discourses on the US-Japan Relationship », in WilliamsC. (dir.) The Political Geography of the New World Order, p.181-209.

Ó Thuatail G., 1996, Critical Geopolitics: The Politics of Writing Global Space, Minneapolis, University of Minnesota, $382 \mathrm{p}$.

Pounds N., 1963, Political Geography, New York, Mc Graw-Hill Book Company.

RothkopfD., 2009, La caste. Les nouvelles élites et le monde qu'elles nous préparent, Paris, Robert Laffont, 330 p. [éd. originale : Superclass. The Global Power Elite and the World They Are Making, 2008, New York, Farrar, Straus and Giroux]

Ratzel F., 1987, La géographie politique. Les concepts fondamentaux, Paris, Fayard, 220 p.

Rioux M., (dir.), 2005, Globalisation et pouvoir des entreprises, Outremont (QB), Athéna/Centre d'études internationales et Mondialisation, 246 p.

Rosière S., 2003, Géographie politique et géopolitique. Une grammaire de l'espace politique, Paris, Ellipses, $320 \mathrm{p}$.

Strange S., 1988, States and Markets, Londres, Pinter.

Strange S., 1996, The Retreat of the State: The Diffusion of Power in the World Economy, Cambridge, Cambridge University Press, XVII-218 p. 
Wolfers A.,1962, Discord and Collaboration: Essays on International Politics, Baltimore, The Johns Hopkins Press, xviii-283 p.

\section{NOTES}

1. La géopolitique classique est ici comprise non pas tellement de façon chronologique (comme la plus ancienne forme de la discipline) mais, thématiquement, comme une discipline praxéologique centrée sur l'interaction État/espace/pouvoir.

2. Comme le soulignait Michel Foucher (1991, pp.33-35), la géopolitique est une "méthode » (une démarche scientifique apparue au XIXe s.), mais aussi une «pratique » (immémoriale) du pouvoir.

3. Le sous-titre de l'édition de 1903 de son ouvrage était: Die geographie der Staaten, des Verkehres und des Krieges, soit « La géographie de l'État, de la circulation et de la guerre ».

4. Selon l'expression structurée par le sociologue Raymond Boudon, cet « individualisme " prône que les phénomènes collectifs doivent être décrits et expliqués à partir des propriétés et des actions de l'individu.

5. « Re-conceptualization of geopolitics in terms of discourse » (O'Tuathail, 1992).

\section{RÉSUMÉS}

Même si l'économie est intégrée à la réflexion géopolitique depuis Friedrich Ratzel au moins, la géopolitique classique accorde une place marginale aux acteurs économiques en tant que tels. Cependant, les mutations contemporaines de l'ordre international (notamment la fin de la guerre de froide et le retrait de l'État consécutif à l'influence de la pensée libérale) ont remis en question cette position. Au-delà de la création de la géoéconomie qui propose un changement de paradigme radical, la géopolitique contemporaine accorde un poids croissant aux firmes multinationales et aux acteurs financiers et remet en question cette perspective "géoréaliste". Cette évolution reflète l'actualité des rapports de pouvoir alors que, de plus en plus, les firmes s'affichent non seulement comme des acteurs majeurs, mais aussi comme des structures concurrençant de plus en plus l'État et l'ordre géopolitique westphalien.

In spite of considering economy (as Friedrich Ratzel did), the classical geopolitical thought did not considered economical agencies with great attention. However the contemporary mutations of political order (and especially the end of 'cold war' and the 'retreat of state' linked with the decisive influence of free market orthodoxy) challenged this position. Beyond the attempt to create geoeconomy - a significant change of paradigm - the contemporary geopolitics considers more carefully multinational companies and financial agencies. This evolution correlates the upto-date evolution of power struggle. Indeed the firms (mostly the multinational companies) act more and more not only as major agencies but like contestants of the state and of the westphalian geopolitical ordrer.

Obgleich die Wirtschaft seit der Zeit Friedrich Ratzel in der geopolitischen Betrachtung integriert ist, gewährt die klassische Geopolitik den wirtschaftlichen Akteuren lediglich eine Nebenrolle. Die heutige Umwandlung der internationalen Ordnung (insbesondere das Ende des Kalten 
Krieges und der Rückzug des Staaten infolge des Einflusses der liberalen Denkweise) hat diese Haltung in Frage gestellt. Außerhalb der Entstehung der "Geowirtschaft", die einen radikalen Paradigmentwechsel bietet, gewährt die heutige Geopolitik eine wachsende Bedeutung zu den internationalen Firmen und zu den finanziellen Akteuren und stellt in Frage diese georealistische Perspektive. Diese Entwicklung spiegelt die Aktualität der Machtverhältnisse wider, während immer mehr die Firmen sich nicht nur als Hauptakteure zur Schau stellen, sondern auch als konkurrierende Strukturen des Staaten und der westphälischen Ordnung.

INDEX

Keywords : epistemology of geopolitics, firms, geopolitics, hegemony, mutinational companies Mots-clés : épistémologie de la géopolitique, firme, géopolitique, hégémonie, multinationales Schlüsselwörter : Epistemologie der Geopolitik, Firme, Geopolitik, Hegemonie, multinationale Konzerne

\section{AUTEUR}

\section{STÉPHANE ROSIÈRE}

Professeur aux Universités de Reims Champagne-Ardenne (France) et Matej Bel (Banska Bystrica, Slovaquie) - Directeur de publication de la revue en ligne L'Espace Politique, http:// espacepolitique.revues.org 